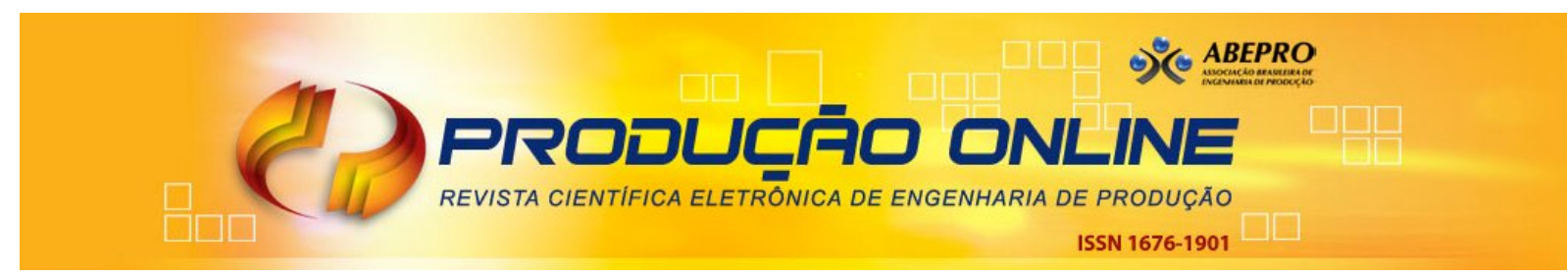

\title{
O USO DA ESTRATÉGIA DE CUSTOMIZAÇÃO EM MASSA EM EMPRESAS DA CONSTRUÇÃO CIVIL
}

\section{THE USE OF THE MASS CUSTOMIZATION PROCESS IN COMPANIES FROM THE CONSTRUCTION SECTOR}

\author{
Diego de Castro Fettermann* E-mail: dcfettermann@gmail.com \\ Rafaella Peixoto Del Pomo de Oliveira* E-mail: rafadelpomo@hotmail.com \\ Giuliano de Almeida Marodin* E-mail: gamarodin@gmail.com \\ *Universidade Federal do Rio Grande do Sul (UFRGS), Porto Alegre, RS
}

\begin{abstract}
Resumo: A estratégia de Customização em Massa (CM) é recomendada para melhorar o atendimento aos requisitos dos clientes e reduzir custos em empresas de manufatura, mas também em setores de baixa escala de produção, como a construção civil. Este artigo tem por objetivo identificar como é realizado o processo de CM em empresas na Construção Civil e compará-lo com a literatura. Os três estudos de caso nos empreendimentos habitacionais permitiram identificar como é aplicada a CM pelas empresas do setor. Os processos de customização aplicados pelas empresas foram analisados de acordo com as propostas mencionadas na literatura. A partir dessa comparação foram identificadas diversas oportunidades de melhoria. As empresas analisadas tem por característica possuírem uma baixa aderência das atividades e técnicas recomendadas pela literatura, sendo que este estudo permitiu aprofundar o conhecimento sobre as oportunidades de melhoria para as empresas do setor.
\end{abstract}

Palavras chave: Customização em Massa. Construção Civil. Agregação de Valor.

Abstract: Mass Customization (MC) is often used to improve customer service and to reduce operational costs in manufacturing companies, and it can also be applied in low-scale contexts, such as construction. This paper aims to assess how construction companies are implementing MC. We compared a framework of MC found in the literature with three case studies and several improvement opportunities were highlighted. As a result, we could conclude that the $M C$ is being poorly used in the construction business and companies can benefit by increasing the implementation of MC tools and techniques.

Keywords: Mass Customization. Construction. Adding Value.

\section{INTRODUÇÃO}

As empresas tendem a aumentar a variedade do portfólio de produtos disponibilizado no mercado como forma de atender a crescente diversidade de necessidades dos clientes (PINE II, 1993). Entretanto, a simples adoção desta estratégia resulta em uma redução do desempenho operacional da empresa, visto 
que um portfólio maior pode resultar no aumento dos custos de manufatura, dos custos indiretos, dos níveis de estoques e dos tempos de produção (SALVADOR et al., 2002). Esse aumento dos custos também está associado ao nível de customização disponibilizado. Geralmente, níveis superiores de customização estão relacionados com maiores custos operacionais, ao mesmo tempo em que viabilizam uma maior variedade de produtos e o atendimento a necessidades mais específicas dos clientes (FOGLIATTO et al., 2012).

A adoção desta abordagem está relacionada à habilidade em disponibilizar produtos customizados por meio de processos produtivos flexíveis com grande volume de produção e a um custo baixo (DA SILVEIRA et al., 2001; TU et al., 2007). Para isto, os clientes devem ser pensados como únicos, sendo indispensável a sua integração ao processo de customização (PEPPERS; ROGERS, 1997; GILMORE; PINE II, 2000). Sievänen (2002) descreve o processo de Customização em Massa (CM) como uma diferenciação de produtos que procura atender às necessidades individuais do maior número possível de clientes. Dessa forma, a CM pode ser compreendida como o desafio de atender às necessidades individuais dos consumidores mantendo a eficiência da manufatura em níveis semelhantes ao da produção em massa (JIAO; TSENG, 2004; KUMAR; PHROMMATHED, 2005). Apesar dos clientes desejarem uma ampla variedade de atributos nos produtos, recomenda-se limitar o conjunto de opções de customização a fim de alcançar economia de escala nos processos de manufatura (KOTHA, 1996). Assim, é importante para a empresa identificar quais são os requisitos mais viáveis a serem customizados e alinhar seu portfólio com o que os clientes mais gostariam de customizar (AHLSTROM; WESTBROOK, 1999).

O emprego da estratégia de CM é mencionada como uma eficiente alternativa para as empresas de manufatura (PINE II, 1993; GILMORE; PINE, 1997; DA SILVEIRA et al., 2001; FOGLIATTO et al., 2012). São verificados diversos casos de aplicação desta estratégia, tais como a fabricação de bicicletas (KOTHA, 1996), Hewlett-Packard (FEITZINGER; LEE, 1997), Dell Computers (RO et al., 2007), móveis modulados (FETTERMANN; ECHEVESTE, 2011), indústria automobilística (BARDACKI; WHITELOCK, 2004; FETTERMANN et al., 2012). O emprego dessa 
estratégia tem se difundido em outros setores além da manufatura, como no desenvolvimento de serviços (VOSS; HSUAN, 2009), em atividades de turismo (CHENG; HAN, 2013) e no desenvolvimento de software (KRUEGER, 2001). Além dessas aplicações, se verifica uma oportunidade de desenvolvimento dessa estratégia na construção civil (SHIN et al., 2008; DUARTE, 2005; HOFMAN et al., 2006), principalmente procurando agregar maior valor ao produto final (ROCHA et al., 2012). O objetivo da CM na construção civil é fornecer uma habitação de qualidade a um custo acessível (DUARTE, 2005), sendo sua aplicação reportada nos mais diferentes mercados, tais como EUA (KENDALL; TEICHER, 2000), GrãBretanha (BARLOW, 1998) e Japão (BARLOW et al., 2003). Além disso, são identificados diversos estudos sobre a aplicação da estratégia de CM na produção de insumos para o setor da Construção Civil (SHIN et al., 2008; HOFMAN et al., 2006; HALMAN et al., 2008; NAHMENS; BINDROO, 2011). Apesar da indicação da $\mathrm{CM}$ na construção civil, existe uma grande variedade de recomendações disponíveis que dificultam o desenvolvimento dessa estratégia na prática das empresas (ROCHA et al., 2012), sendo esta também uma dificuldade na área de manufatura (FETTERMANN; ECHEVESTE, 2014).

A partir dessa dificuldade, este trabalho tem por objetivo identificar como é realizado o processo de $\mathrm{CM}$ na Construção Civil e compará-lo com o recomendado pela literatura. A partir dessa comparação pretende-se identificar oportunidades de melhoria na forma como as empresas de Construção Civil empregam a estratégia de CM. Este estudo está limitado a empresas que aplicam a estratégia de CM na construção civil com empreendimentos na região sul da Bahia. O trabalho está estruturado com uma introdução que aborda o tema de $\mathrm{CM}$, tanto no geral quanto na construção civil. A revisão de literatura apresenta os temas acerca da estratégia de $\mathrm{CM}$, as etapas do processo de aplicação da CM e seus desdobramentos na construção civil. A terceira seção apresenta a descrição do método de pesquisa utilizado. Na quarta seção estão os resultados, apresentando primeiramente o estudo de caso da aplicação da CM nas empresas estudadas seguido do comparativo entre as recomendações da literatura e o aplicado pelas empresas 
analisadas. Por fim, são apresentadas na quinta seção as considerações finais e as oportunidades de pesquisa.

\section{REVISÃO DE LITERATURA}

Nesta seção será apresentado o referencial teórico para a condução do presente trabalho, o qual contempla: variedade de produtos, agregação de valor pela customização tanto para a empresa como para o consumidor, o processo de aplicação da CM e, por fim, aplicações da CM na construção civil.

\subsection{Customização em Massa}

A variedade de produto é definida como a diversidade de produtos que uma empresa de manufatura fornece para o mercado (ULRICH, 1995). A CM tem o objetivo de atender a grande quantidade de clientes e o mesmo tempo tratá-los individualmente (DAVIS, 1987), sendo recomendada para mercados relativamente grandes e ao mesmo tempo atendendo as necessidades individuais dos consumidores (BLECKER et al., 2003). Como forma de atingir essa maior variedade de produtos recomenda-se o desenvolvimento da capacidade de projetar, produzir e comercializar os produtos de alta qualidade em um curto espaço de tempo e com um custo acessível para os clientes (TSENG; JIAO, 2007). Para tanto, as empresas devem investir em padronização dos produtos ao mesmo tempo em que promovem a variedade das características valorizadas pelos clientes (PINE II, 1993).

Apesar de a variedade ser valorizada pelos clientes, o excesso de opções também pode tornar o processo de customização confuso (mass confusion), reduzindo a vantagem competitiva da CM (HUFFMANN; KAHN, 1998). Assim, o processo de customização deve identificar quais atributos dos produtos estão relacionados com as necessidades dos clientes e selecionar quais customizações serão disponibilizadas nesses atributos para atender estas necessidades (SQUIRE et al., 2004). 
No paradigma da produção em massa, os clientes estavam dispostos a limitar suas escolhas de produto apenas para o que estava disponível no mercado (PINE II, 1993). Com o passar do tempo os clientes aumentaram seu padrão de exigências e estão menos dispostos a limitar sua escolha aos produtos padronizados (on the shelf) (TSENG; JIAO, 2007). A partir da interação entre a empresa e o cliente durante o projeto do produto se viabiliza a configuração do produto de acordo com as necessidades do cliente, e com isso uma agregação de valor e uma maior disposição do cliente em pagar por essa opção (FRANKE et al., 2010; ULRICH, 2011). Esta disposição para pagar a mais por um produto customizado tende a viabilizar uma maior margem de lucro para as empresas (ROBERT; MEYER, 1991), além do aumento do nível de satisfação do cliente (JIAO; TSENG, 2004).

Um projeto customizado não pode disponibilizar todas opções para o cliente, pois uma variedade excessiva de opções tende a resultar em altos custos em projeto, produção, estoque e logística (DA SILVEIRA et al., 2001). A partir disso, a indústria da construção civil deve selecionar e desenvolver um conjunto de alternativas viáveis para a customização pelo cliente. Ao definir o que pode ser personalizado, a empresa pode planejar os processos que serão necessários para fornecer a customização de forma eficiente (ROCHA et al., 2012). A solução para este conjunto de opções deve considerar a abrangência do mercado e as características mais valorizadas pelos clientes.

Os clientes tornam-se dispostos a pagar mais por produtos que sejam exatamente como eles desejam. O "foco no cliente" é um termo reconhecido como a capacidade de uma organização para atender as necessidades do cliente com alto nível de satisfação do mesmo (BARLOW; OZAKI, 2003). Esta preferência dos clientes é formada baseando-se em percepções de valores de um produto e no resultado de uma avaliação de vantagens e desvantagens quando relacionados à sua aquisição e utilização (ZEITHAML, 1988). Os atributos são considerados como variáveis que afetam a decisão de compra de um cliente, tais como características relacionadas à funcionalidade do produto, qualidade, marca, design etc. (LIHRA et al., 2012). No entanto, a diferença de preço que os clientes possam aceitar é limitada e depende do valor percebido pela personalização (HUFFMANN; KAHN, 
1998; HART, 1995). Além disso, o tempo de entrega dos produtos customizados não pode ser muito diferente dos produtos padronizados, pois os clientes podem atribuir uma importância considerável ao prazo de entrega no momento de decidir a sua compra (SQUIRE et al., 2004; LIHRA et al., 2012). Pine II (1993) menciona que o valor do cliente é resultado de um equilíbrio entre qualidade, custo e tempo de entrega. Na visão da empresa, além da vantagem de agregação de valor pela possibilidade de customização, o processo de customização permite a iteração com o cliente, viabilizando o acesso à informações de mercado e a fidelização do cliente (PILLER, 2004; FOGLIATTO et al., 2012).

\subsection{Processo de Customização em Massa}

Verifica-se a necessidade de um fluxo de informações e atividades para tornar viável a customização dos produtos (KUMAR; PHROMMATHED, 2005). Este fluxo de informações e atividades incorpora desde a busca de conhecimentos sobre o comportamento do mercado até sua tradução em especificações do projeto, produção e entrega do produto ao cliente (FETTERMANN; ECHEVESTE, 2011). De forma a compreender como é realizada a interação entre o cliente e a produção durante o processo de customização foi realizada uma revisão das recomendações da literatura (GILMORE; PINE II, 1997; DURAY et al., 2000; DA SILVEIRA et al., 2001; ROYER, 2007, FETTERMANN; ECHEVESTE, 2011), que foi estruturada de acordo com as etapas do processo de customização (FIGURA 1).

Figura 1- Processo de customização de produtos

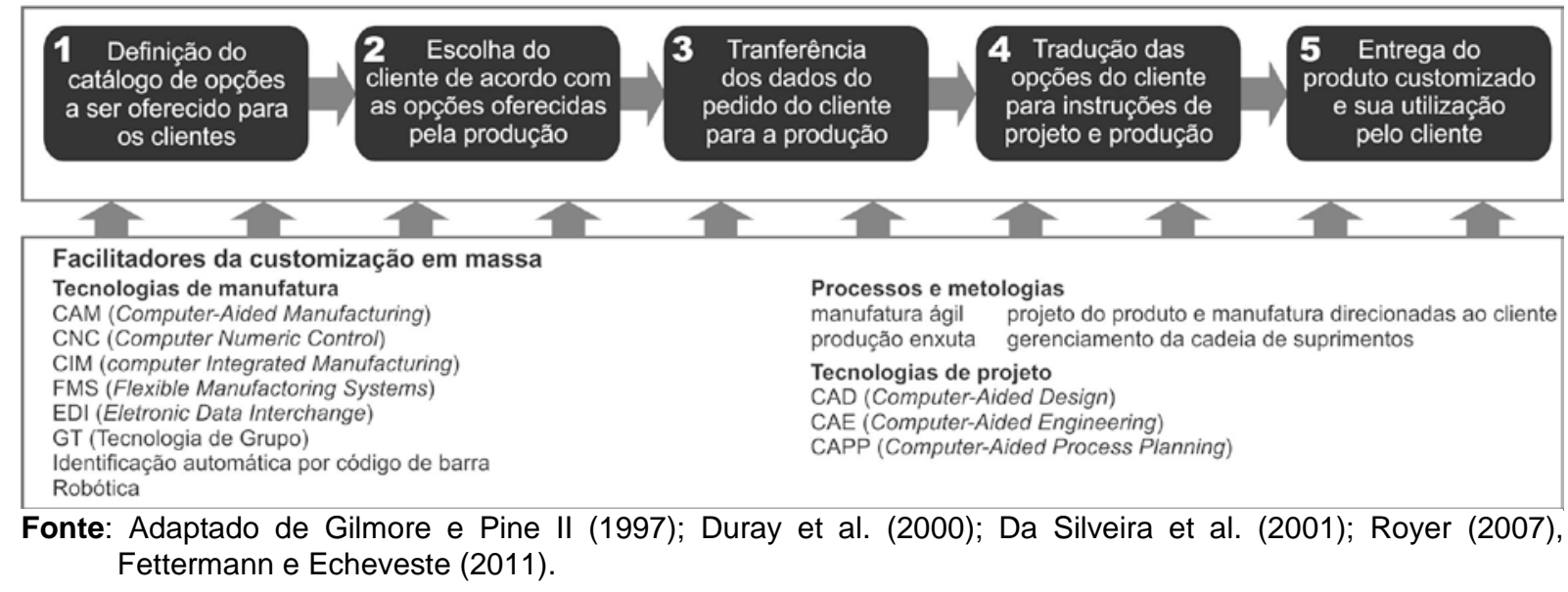

Revista Produção Online, Florianópolis, SC, v.15, n. 1, p. 135-162, jan./mar. 2015. 
A primeira etapa define quais opções serão oferecidas para serem customizadas (DA SILVEIRA et al., 2001). Nesta etapa, recomenda-se verificar a necessidade da customização do produto, a viabilidade para customizá-lo, além da disponibilidade de tecnologia e logística para as atividades de produção (DA SILVEIRA et al., 2001). Também é recomendado que a construtora interprete as necessidades do cliente para desenvolver as opções de customização. A possibilidade de disponibilização de configuradores de produto têm sido mencionada como uma alternativa para captar as necessidades do cliente e apresentá-las na forma de um projeto customizado (FETTERMANN et al., 2012; FOGLIATTO et al., 2012).

$\mathrm{Na}$ segunda etapa é considerada a forma como o cliente definirá suas escolhas pelas alternativas que são oferecidas para customização. Um dos fatores determinantes para o sucesso desta etapa é a facilidade do cliente em mapear suas necessidades e traduzi-las no projeto do produto customizado (DURAY; MILLIGAN, 1999; OGAWA; PILLER, 2006). Na construção civil, esta etapa pode ocorrer por meio de um configurador que possibilite o cliente simular e escolher quais opções ele prefere. Este processo também pode ser realizado com o auxílio de um projetista capaz de suportar o cliente durante a execução do projeto do produto customizado. Além disso, a experiência de projeto do cliente durante o processo de customização é considerada uma importante variável na agregação de valor ao produto customizado (FRANKE et al., 2010; MERLE et al., 2010; MOREAU, 2011). A utilização de configuradores de produto baseados na internet também tem sido utilizada para este objetivo (HELLANDER; JIAO, 2002; FETTERMANN et al., 2012)

Na terceira etapa é realizada a transferência das opções de customização do cliente desde o ponto de venda para a produção. A automatização desta transferência pode contribuir para a execução desta etapa. Porém, na construção civil este procedimento ainda tende a ser realizado manualmente, resultando em uma sobrecarga de trabalho pelo excesso de contatos e seus desdobramentos, tais como, cálculos relacionados ao valor que será acrescido depois das alternativas escolhidas pelo cliente (ARAUJO FILHO; GOMES, 2010). Alternativas utilizadas em 
outros setores, como de móveis modulados podem contribuir neste propósito. As empresas deste setor utilizam o próprio software CAD utilizado no projeto customizado do produto $\left(\mathrm{PROMOB}^{\circledR}\right)$ para identificar os módulos e encaminhar a lista de produtos para a produção por meio da utilização de EDI (Eletronic Data Interchange) (FETTERMANN; ECHEVESTE, 2011).

A quarta etapa, tradução das opções do cliente para instruções de projeto e manufatura inicia-se no momento em as especificações do projeto customizado são interpretadas na empresa e transformadas em instruções de manufatura (DA SILVEIRA et al., 2001). Para tanto, existe uma variedade de opções, tais como modelos para otimização de custos de manufatura (DU et al., 2003), a utilização das tecnologias CAD e CAM (KOTHA, 1996), além de outras tecnologias de manufatura, tais como FMS (Flexible Manufacturing System). Entre essas tecnologias de manufatura está a utilização de sistemas capazes de automatizar a comunicação entre sistemas de projeto e manufatura, como no caso de móveis modulados. Algumas empresas deste setor possuem sistemas capazes de integrar as especificações do projeto customizado, desenvolvidas no software CAD PROMOB ${ }^{\circledR}$ e traduzí-las de forma automática na programação necessária para os centros de usinagem CNC (Computer Numeric Control) possam produzir a especificação customizada do produto (FETTERMANN; ECHEVESTE, 2011). Além disso, arranjos de produção mais flexíveis, como células de manufatura, também podem contribuir para a produção de itens customizados (TSENG; JIAO, 1996).

Por fim, a quinta etapa, na qual consiste na entrega e utilização do produto pelo cliente. Nessa etapa, podem-se incluir as customizações que são realizadas na entrega do produto e no uso da edificação (LIZARRALDE, 2011), como a disponibilização de serviços (VIDOR et al., 2013). Nesse caso, além do projeto de adaptações e ou mobiliário após a entrega, também são comuns solicitações de alterações de pontos elétricos, adaptações da rede hidráulica, sistema de iluminação, além de trocas de piso e alvenarias. Normalmente, essas customizações acontecem sem o conhecimento e consentimento da construtora, podendo resultar em perda de garantia e danos a estrutura da construção. 


\subsection{Aplicações da CM na Construção Civil}

A aplicação da estratégia de CM na construção civil tem sido recomendada como forma de atender as exigências de clientes, geralmente de maior poder aquisitivo, em customizar a habitação de acordo com seus critérios individuais, após o recebimento do mesmo (BARLOW et al., 2003). A partir desta percepção, as construtoras possibilitaram aos compradores opções de customização desde a fase inicial de construção (ARAUJO FILHO; GOMES, 2010). Assim, a personalização de habitações tem sido uma estratégia de negócio importante para promover o mercado imobiliário (BARLOW; OZAKI, 2003), desenvolvendo uma especificidade de aplicação na indústria na construção civil (BARLOW et al., 2003). A utilização da CM permite reduzir os custos de produção por meio de economias de escopo, baseada na padronização de componentes, enquanto atende mais precisamente as necessidades individuais de cada cliente (NOGUCHI; VELASCO, 2005). Existem diversos fatores determinantes para a decisão de compra por parte do cliente, sendo que a maioria deles independe do projeto em sí, tais como: localização, características do ambiente, preço, etc. Ainda assim, é vendido ao consumidor o ambiente interior, sendo que este pode ser customizado de acordo as suas necessidades (BRANDÃO, 2002). Para isso, os empreendimentos habitacionais disponibilizam uma grande variedade de opções para customização interna, tais como acabamentos, instrumentos de automação, serviços pós-ocupação entre outros (BARLOW et al., 2003).

De acordo com as características do desenvolvimento do projeto, as empresas da construção civil podem ser classificadas em três diferentes categorias: (i) produção, (ii) semi-customizadas e (iii) customizadas (NOGUCHI; VELASCO, 2005). As empresas categorizadas em produção estão direcionadas as vantagens de escala de produção, produzindo unidades padronizadas em menor tempo e custo. As semi-customizadas analisam a viabilidade de customização dos atributos da unidade e disponibilizam um conjunto de opções viáveis de serem customizadas pelo cliente de acordo com suas necessidades. As customizadas oferecem liberdade total de customização ao cliente, criando projetos originais e únicos (NOGUCHI; 
VELASCO, 2005). As empresas que trabalham com projetos da categoria semicustomizados são as que possuem maior possibilidade de empregar a estratégia de CM.

A customização na construção civil deve considerar tanto produtos como serviços (NOGUCHI; VELASCO, 2005). Os produtos podem ser considerados como atributos determinados por componentes integrantes da unidade, como acabamentos e especificações de materiais. Enquanto isso, o projeto e a construção são considerados como serviços que podem ser customizados (NOGUCHI; VELASCO, 2005).

\section{MÉTODO}

A presente pesquisa procura investigar o processo de customização de produtos utilizado pelas empresas de construção civil de empreendimentos habitacionais. Esta pesquisa pode ser caracterizada como exploratória, visto que tem por objetivo compreender o fenômeno e tornar o conhecimento mais explícito (GIL, 2002). Como forma de atingir esse objetivo foi utilizada a abordagem qualitativa, sendo este tipo de abordagem indicada para compreender o fenômeno segundo a perspectiva dos participantes da situação estudada (NEVES, 1996). Para isso, foram realizadas entrevistas de profundidade seguindo um questionário semiestruturado (RIBEIRO; MILAN, 2004) com representantes das empresas envolvidas no processo de customização das empresas. O questionário utilizado foi construído procurando identificar a forma como é realizada cada uma das etapas do processo de CM (FIGURA 1) empregado pelas empresas de construção civil com projetos semi-customizados ou customizados localizados no sul da Bahia. Desta forma, são formuladas diversas questões para identificar a forma como são executadas cada uma das etapas do processo de customização. Após a finalização do questionário (ANEXO A), foi realizado um pré-teste. Segundo Gil (2002), este procedimento visa garantir que o instrumento atinja os objetivos propostos evitando erros de compreensão ou entendimento por parte dos respondentes. O pré-teste do questionário foi realizado com dois especialistas da área de construção civil, 
posteriormente as sugestões coletadas foram incorporadas ao questionário. Assim como o questionário, os resultados são analisados e apresentados procurando descrever os procedimentos utilizados pelas empresas para aplicação da estratégia de Customização em Massa. Dessa forma, os resultados obtidos são apresentados de forma descritiva e posteriormente são comparados com as proposições identificadas na literatura. A partir dessa comparação, são identificadas possibilidades de melhoria para as empresas analisadas.

O objeto de estudo são empresas de construção civil na região sul da Bahia que atuam no segmento de empreendimentos habitacionais e que desenvolvem projetos da categoria semi-customizados ou customizados. A partir disso, foram identificadas 12 construtoras na região com empreendimentos habitacionais com projetos dessa categoria. Essas empresas tem por característica possuir médio porte e desenvolver pelo menos duas construções habitacionais de forma simultânea. Entre essas empresas, foram selecionadas três de acordo com a sua disponibilidade em participar da pesquisa. Nessas empresas, foram entrevistados profissionais com conhecimento sobre o processo de projeto, construção e venda das unidades. Cada uma das três entrevistas realizadas teve duração aproximada de 90min e todas tiveram o seu conteúdo transcrito.

\section{RESULTADOS}

Os resultados são apresentados em três subseções. Primeiramente, são apresentadas cada uma das empresas participantes da pesquisa, identificando suas características, atuação e porte. Posteriormente, é abordada a forma como cada uma das empresas atua em cada etapa do processo de customização. Por fim, é realizado um comparativo entre as recomendações da literatura em cada uma das etapas do processo de customização e o aplicado pelas empresas, com o objetivo de identificar oportunidades de desenvolvimento para as empresas do setor.

\subsection{Descritivo das empresas}


Para a aplicação do questionário, a Empresa A disponibilizou um funcionário responsável pelo atendimento ao cliente, com formação de engenharia de produção. A Empresa B disponibilizou um corretor responsável pelas vendas dos terrenos e com conhecimento amplo sobre o processo de customização e a Empresa C disponibilizou um engenheiro civil responsável por uma das obras da empresa (Tabela 1).

Tabela 1 - Descritivo das características das empresas analisadas

\begin{tabular}{lccc}
\hline & Empresa A & Empresa B & Empresa C \\
Quantidade funcionários & 230 & 300 & 178 \\
\hline Tempo de atuação como construtora & 10 anos & 40 anos & 28 anos \\
Região de atuação & Ilhéus e Itabuna & Ilhéus e Salvador & Itabuna \\
Qtd de pavimentos das edificações residenciais & Até 18 pav. & Até 2 pav. & Até 20 pav. \\
$\begin{array}{l}\text { Qual tipo de customização (semi } \\
\text { customizada/customizada) }\end{array}$ & Semi Customizada & Customizada & Semi Customizada \\
Fonte: O Autor (2014) & & &
\end{tabular}

\subsection{Processo de customização}

No processo de customização, há o ponto de desacoplamento (DP), o qual consiste no momento em que as especificações dos clientes começam a ser consideradas no projeto do produto (ARAUJO FILHO; GOMES, 2010), também denominado do momento de integração do cliente (DURAY et al., 2000). A Tabela 2 apresenta o DP de acordo com as etapas do projeto da habitação (ARAUJO FILHO; GOMES, 2010) nas três empresas estudadas. A Empresa A e a Empresa C começam a incluir as especificações do cliente na etapa de demarcação das alvenarias, possibilitando a customização das mesmas de acordo com as necessidades dos clientes. As atividades posteriores a esta etapa podem ser alteradas em razão da demanda do cliente. A Empresa B inclui as especificações do cliente a partir da aquisição do terreno, dando-lhes total liberdade para elaborar o seu projeto. 
Tabela 2 - Comparativo do ponto de desacoplamento (DP) entre as empresas estudadas

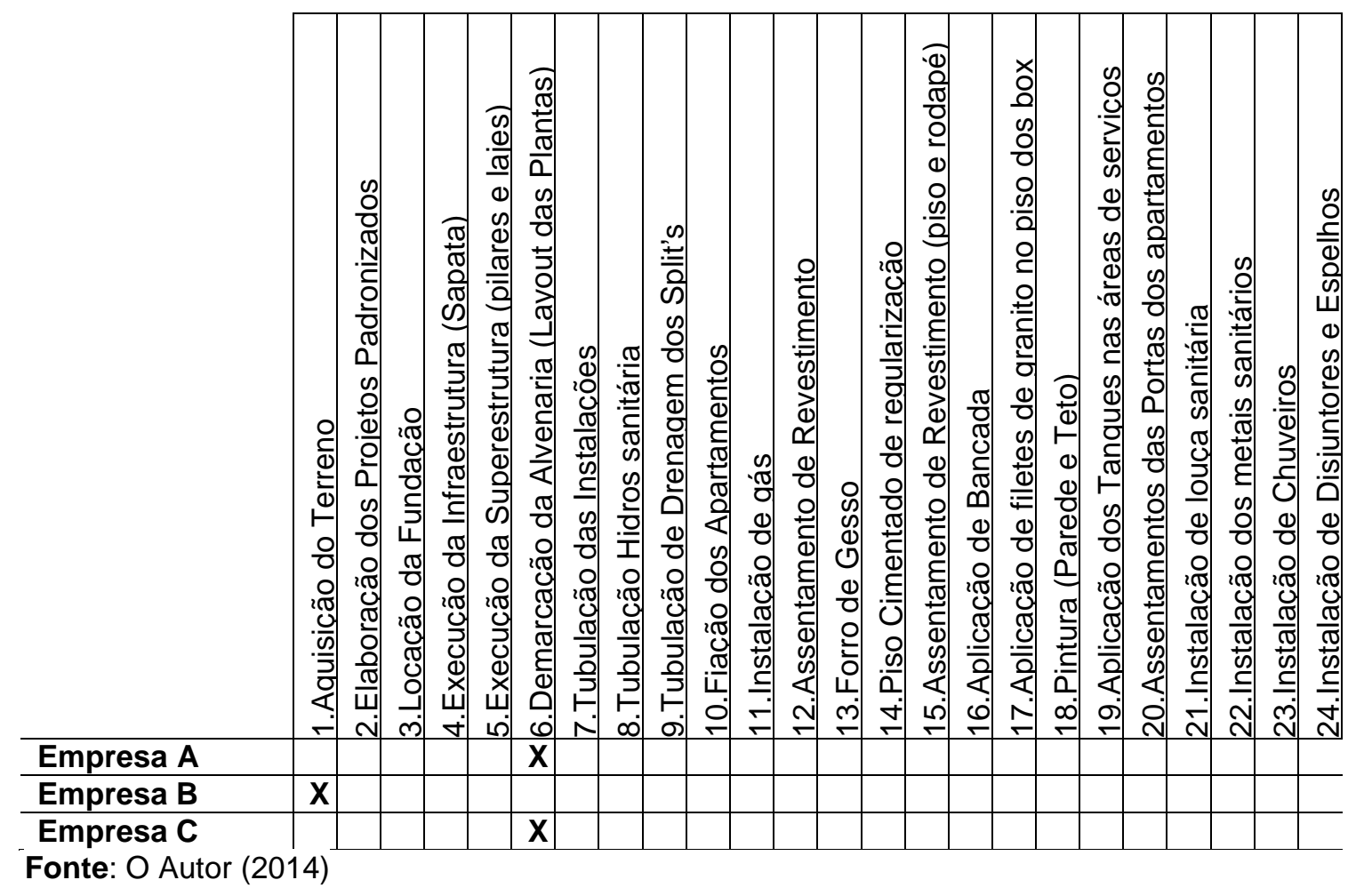

\subsubsection{Definição do catálogo de opções}

Anteriormente, a Empresa A disponibilizava total liberdade para o cliente alterar o acabamento de piso, paredes, disposição dos aparelhos sanitários e alvenarias mediante a submissão de um projeto realizado por um profissional habilitado. Entretanto, esse procedimento está sendo abandonado pela empresa. A Empresa A passou a disponibilizar em seus empreendimentos habitacionais alternativas de planta baixa, em que o cliente pode selecionar a disposição das alvenarias de acordo com as opções pré-estabelecidas. A empresa utiliza a experiência adquirida nos empreendimentos anteriores para estabelecer as opções de planta baixa. A Empresa B é responsável pelas vendas dos terrenos e oferece total liberdade ao cliente para a contratação de engenheiros e arquitetos de modo a customizar a unidade. As únicas limitações para customização estão restritas ao regime urbanístico estabelecido para a localidade, tais como recuos, altura da edificação, taxa de ocupação e índice de aproveitamento. A Empresa C define o 
catálogo de opções de customização de acordo com as características do empreendimento e experiências anteriores. O cliente pode selecionar as opções préestabelecidas para customizar o interior da edificação desde que não interfira na estrutura da edificação. Entre as três empresas pesquisadas, não foi possível identificar a utilização de algum método e técnica estruturada para prospectar ou definir quais atributos da edificação serão disponibilizados para a customização.

\subsubsection{Escolha do cliente de acordo com as opções estabelecidas}

Segundo o engenheiro da Empresa A, no histórico da construtora, a alteração da localização das alvenarias consiste na customização mais solicitada pelos clientes, seguida por alterações de pontos elétricos, pontos hidráulicos e de acabamento de piso. Na Empresa A, as alternativas de customização acontecem mediamente a apresentação de um projeto executivo por um profissional habilitado. Quaisquer solicitações de customização incluem a análise sobre sua viabilidade técnica e o seu efeito no custo do produto. Na Empresa B, o cliente tem total liberdade de customização na unidade. São oferecidos alguns projetos de unidades que podem ser construídas, podendo o cliente utilizar este projeto igual ou totalmente diferente, desde que respeite o regime urbanístico estabelecido. Apesar de disponibilizar liberdade para a customização, o projeto da habitação deve ser realizado por profissional habilitado, sendo que sua execução deve ser realizada pela empresa, mediante avaliação de viabilidade técnica e seu efeito no custo da unidade. A Empresa C determina um prazo aos proprietários para realizar a customização da unidade, sendo que esta customização é realizada baseada em um conjunto de opções de atributos disponibilizados pela construtora. A partir da combinação dos atributos selecionados para a customização é realizado um novo projeto executivo. Em todas as empresas a realização do projeto executivo da customização da unidade é realizado por profissionais externos à empresa. 


\subsubsection{Transferência dos Dados dos Pedidos do Cliente para a Produção}

Esta transferência de informações acontece manualmente nas empresas estudadas, sendo realizada por meio de solicitações em papel acompanhadas de projeto executivo realizado por profissional habilitado ou por via eletrônica, com a digitalização dos documentos. No caso da Empresa A e da Empresa C, a transferência dos dados é realizada principalmente da forma digitalizada. $\mathrm{Na}$ Empresa B, em razão do maior nível de customização disponibilizado, o projeto de customização é transferido diretamente pelo profissional responsável pelo projeto, ocorrendo principalmente de forma digitalizada.

\subsubsection{Tradução dos pedidos do cliente para instruções de projeto e produção}

Segundo o engenheiro representante da Empresa A, qualquer solicitação de customização deve passar por uma análise de viabilidade técnica. Esta análise, principalmente em relação ao projeto arquitetônico, elétrico e hidráulico, é realizada pelo setor de projetos da empresa. Posteriormente, esse projeto de customização compatibilizado é aprovado pelo cliente e disponibilizado no momento de sua execução no empreendimento. O engenheiro da Empresa A menciona que a variabilidade de solicitações dos clientes tende a prejudicar a produtividade da obra. Além disso, diversas solicitações de customização dos clientes, tais como alterações na paginação do piso e paredes tem sido negada pela empresa em razão da complexidade em executar com qualidade esta customização. No caso da Empresa B, por ser de total customização, não há um modelo pré definido de projeto. Então, o custo da mão de obra e produtividade só serão definidos após a escolha do cliente. O cliente fará o projeto de acordo com suas necessidades com o profissional de sua escolha e o engenheiro da empresa realizará a análise e tradução das especificações para instruções de produção. A Empresa C passa por situação semelhante a Empresa $\mathbf{A}$, na qual é realizada, primeiramente, uma compatibilização do projeto customizado com o projeto original do empreendimento. Após esta 
compatibilização, o projeto customizado fica disponível para a execução da produção.

\subsubsection{Entrega do produto customizado e sua utilização pelo cliente}

Segundo o engenheiro da Empresa A, apesar de a empresa não disponibilizar nenhum serviço pós ocupação, foi mencionada a demanda de clientes por este tipo de serviço e a oportunidade de negócio para a empresa. A Empresa B, apesar de oferecer um maior nível de customização, também não disponibiliza nenhum serviço pós ocupação. A Empresa C disponibiliza a possibilidade de customização de alguns atributos após a entrega da unidade mediante avaliação da solicitação.

\subsection{Oportunidades de desenvolvimento}

A partir da investigação sobre o processo de customização aplicado pelas empresas de construção civil foi realizada uma comparação com as recomendações mencionadas pela bibliografia para cada etapa do processo de CM (TABELA 3). Nessa comparação foram identificadas que diversas das recomendações mencionadas na literatura não são aplicadas nas empresas estudadas. Com isso, foram identificadas oportunidades de desenvolvimento para as empresas analisadas em cada uma das etapas do processo de customização. Nenhuma das oportunidades mencionadas teve sua avaliação econômica realizada. 


\begin{tabular}{|c|c|c|c|}
\hline Recomendação & Autores & $\begin{array}{c}\text { Etapa } 1 \text { - Definição do catá } \\
\text { Casos estudados }\end{array}$ & logo de opções \\
\hline $\begin{array}{l}\text { Identificar itens } \\
\text { customizáveis de maior } \\
\text { capacidade de agregação } \\
\text { de valor. }\end{array}$ & $\begin{array}{l}\text { Da Silveira et al. (2001); } \\
\text { Tseng et al. (2010); Jiao } \\
\text { et al. (2003); }\end{array}$ & $\begin{array}{l}\text { Avaliação das solicitações } \\
\text { de customização dos } \\
\text { empreendimentos } \\
\text { anteriores. }\end{array}$ & $\begin{array}{l}\text { O desenvolvimento de uma família de produto, com os itens } \\
\text { customizáveis na edificação mais valorizados pelo cliente permite a } \\
\text { empresa uma maior padronização das opções mantendo vantagens de } \\
\text { escala de produção. }\end{array}$ \\
\hline $\begin{array}{l}\text { Estudo de segmentação de } \\
\text { mercado. }\end{array}$ & $\begin{array}{l}\text { Drejer e Gudmundsson } \\
\text { (2002); Jiao et al. (2003); } \\
\text { Grenci e Watts (2007) }\end{array}$ & $\begin{array}{l}\text { Não é realizado pelas } \\
\text { empresas }\end{array}$ & $\begin{array}{l}\text { O estudo de segmentação de mercado pode contribuir para as empresas } \\
\text { disponibilizarem um conjunto de itens customizáveis com maior } \\
\text { capacidade de agregar valor diferente para cada segmento de } \\
\text { mercado/empreendimento }\end{array}$ \\
\hline $\begin{array}{l}\text { Integrar o processo de } \\
\text { vendas ao projeto de } \\
\text { novos empreendimentos. }\end{array}$ & $\begin{array}{l}\text { Da Silveira et al. (2001); } \\
\text { Jiao et al. (2003); } \\
\text { Helander e Jiao (2002) }\end{array}$ & $\begin{array}{l}\text { Não é realizado de forma } \\
\text { sistemática pelas } \\
\text { empresas }\end{array}$ & $\begin{array}{l}\text { O contato direto com o cliente durante o processo de vendas é uma } \\
\text { importante fonte de informações do comportamento do mercado, } \\
\text { podendo ser utilizada na definição dos itens customizáveis nos próximos } \\
\text { empreendimentos. }\end{array}$ \\
\hline $\begin{array}{l}\text { Definir um portfólio de } \\
\text { atributos customizáveis } \\
\text { mais otimizado. }\end{array}$ & $\begin{array}{l}\text { Meyer e Lehnard, (1997); } \\
\text { Bare e Cox (2008) }\end{array}$ & $\begin{array}{l}\text { Avaliação das solicitações } \\
\text { de customização dos } \\
\text { empreendimentos } \\
\text { anteriores. }\end{array}$ & $\begin{array}{l}\text { Identificar itens de customização que podem ser padronizados entre os } \\
\text { diferentes empreendimentos da empresa, obtendo maiores vantagens de } \\
\text { escala de produção. }\end{array}$ \\
\hline \multirow[t]{2}{*}{$\begin{array}{l}\text { Utilização de métodos de } \\
\text { padronização de } \\
\text { componentes } \\
\text { (comunalização) }\end{array}$} & $\begin{array}{l}\text { Simpson (2004); Jiao e } \\
\text { Tseng (2000); Robertson } \\
\text { e Ulrich (1998) }\end{array}$ & $\begin{array}{l}\text { Não é realizado pelas } \\
\text { empresas }\end{array}$ & $\begin{array}{l}\text { A utilização de métodos formais de padronização de componentes } \\
\text { contribui para reduzir a quantidade de itens (Part Numbers e SKU's) a } \\
\text { serem gerenciados pela empresa. }\end{array}$ \\
\hline & \multicolumn{3}{|c|}{ Etapa 2 - Escolha do cliente de acordo com as opções estabelecidas } \\
\hline $\begin{array}{l}\text { Sistema de configurador } \\
\text { online para realizar a } \\
\text { customização da unidade }\end{array}$ & $\begin{array}{l}\text { Franke e Piller (2003); } \\
\text { Fogliatto e Da Silveira } \\
\text { (2008); Franke et al. } \\
\text { (2010) }\end{array}$ & $\begin{array}{l}\text { Não é realizado pelas } \\
\text { empresas }\end{array}$ & $\begin{array}{l}\text { A possibilidade de o próprio cliente realizar o projeto de customização é } \\
\text { mencionada como um fator de agregação de valor ao produto e pode ser } \\
\text { suportada pelo configurador de produto. }\end{array}$ \\
\hline $\begin{array}{l}\text { Disponibilizar serviços } \\
\text { associados ao produto }\end{array}$ & $\begin{array}{l}\text { Jiao et al. (2003); Tseng e } \\
\text { Jiao (2007) }\end{array}$ & $\begin{array}{l}\text { Não é realizado pelas } \\
\text { empresas }\end{array}$ & $\begin{array}{l}\text { A disponibilização de projetos auxiliares para a customização das } \\
\text { habitações tais como serviços de projeto arquitetônico, projetos } \\
\text { auxiliares, projeto de mobiliário e decoração podem contribuir para } \\
\text { agregar valor ao produto. }\end{array}$ \\
\hline
\end{tabular}

Revista Produção Online, Florianópolis, SC, v.15, n. 1, p. 135-162, jan./mar. 2015. 
Desenvolver um suporte

ao processo de

configuração (Decision

Support System)

Funcionalidade

tridimensional no modulo do produto

Utilização de sistemas ED para a comunicação entre o ponto de venda e a produção

\section{Células de Manufatura}

Utilização de sistemas para otimização de custos da customização

Genérico da matriz BOM (Bill of Material) (GBOM)

Disponibilização de serviços de customização pós ocupação
Tseng e Jiao (1996);

Helander e Jiao (2002); N Não é realizado pelas

Salvador e Forza (2004); empresas

Grenci e Watts(2007)

empresas
Dai et al. (2006) Não é realizado pelas

Apresentar ao cliente um procedimento para realizar a customização do imóvel pode contribuir para o processo de customização, suportando o cliente durante a seleção das opções.

Ajuda os clientes a terem uma visão mais ampla das suas opções de customização, visualizando os produtos antes da montagem ou fabricação do mesmo.

\section{Etapa 3 - Transferência dos dados dos pedidos do cliente para a produção}

Da Silveira et al. (2001) Não é realizado pelas empresas

Um sistema integrado entre o setor de projetos e a produção pode proporcionar maior agilidade no processo produtivo da empresa.

\section{Etapa 4 - Tradução dos pedidos do cliente para instruções de projeto e produção}

O conceito de célula de manufatura pode ser transferido para a área de projetos customizados, em que cada célula é responsável pelo projeto de customização de um ambiente específico da habitação, padronizando soluções de customização. empresas

Não é realizado pelas

empresas

Du et al. (2003)

Zhang et al. (2005)

Não é realizado pelas empresas

conhecimento em relação à variedade de produtos é organizado e há um processo mestre (MP) que tem um conhecimento sobre a variedade do processo. São gerados um planejamento de produção e matrizes correspondentes para gerenciar as relações entre GBOM e MP.

Também contribui para a análise das viabilidade da customização.

\section{Etapa 5 - Entrega do produto customizado e sua utilização pelo cliente}

A disponibilização de serviços de customização, tais como alterações de Realizado parcialmente pela Empresa C. piso, alvenarias, acabamentos e serviços elétricos. Além disso, o serviço de projeto da customização após a ocupação do produto e durante sua etapa de uso também é uma possibilidade para as empresas.

Fonte: O Autor (2014) 


\section{CONSIDERAÇÕES FINAIS}

Este trabalho teve por objetivo identificar como é realizado o processo de Customização em Massa na Construção Civil e compará-lo com o recomendado pela literatura. Para tanto, foi realizado um estudo de caso em três diferentes empresas construção civil da região sul da Bahia para identificar diferenças entre os métodos e técnicas aplicados pelas empresas e o recomendado pela literatura como forma de identificar oportunidades de melhoria para as empresas do setor. Devido à abrangência da $\mathrm{CM}$, foi possível identificar várias práticas empregadas em outros setores que podem ser adaptadas e ter resultados satisfatórios para a CM na construção civil. Os resultados obtidos identificaram diversas oportunidades de melhoria, principalmente durante as duas primeiras etapas do processo de customização. Essas etapas tem por função identificar as possibilidades de customização e apresentá-las ao cliente. A maior parte das oportunidades de melhoria identificadas para estas etapas tem por característica não necessitar investimentos significativos para sua implementação. Além disso, tendem a utilizar informações já disponíveis na empresa ou de fácil obtenção a partir de sua base de clientes. A partir disso, a coleta de informações do mercado obtidas principalmente durante o processo de vendas e a utilização dessas informações de forma sistematizada no processo de projeto do empreendimento tendem a contribuir para o desenvolvimento da estratégia de CM pelas empresas analisadas.

Dentre as três construtoras em que se realizou a análise, foi possível identificar que a quantidade de métodos e técnicas utilizadas ainda é baixa. Ainda assim, os poucos métodos e técnicas utilizados são aplicados de forma não estruturada e sistematizada, reduzindo as vantagens de sua aplicação. A partir disso, foram identificadas diversas oportunidades de desenvolvimento para as empresas do setor, tais como: estudos de segmentação de mercado, sistemas de configurador online e disponibilização de serviços pós-ocupação. A CM na construção civil ainda pode ser muito explorada e aperfeiçoada, trazendo novas tecnologias, principalmente, para o tratamento e posterior utilização das informações recebidas pelos clientes no processo de CM nas unidades habitacionais. Como 
recomendação para trabalhos futuros, sugere-se a aplicação deste diagnóstico em empresas de construção civil de outras regiões do país e a comparação dos resultados obtidos.

\section{REFERÊNCIAS}

ÅHLSTRÖM, P.; WESTBROOK, R. Implications of mass customization for operations management: an exploratory survey. International Journal of Operations \& Production Management, v.19, n. 3, p. 262-275, 1999. http://dx.doi.org/10.1108/01443579910249705

BARDAKCI, A.; WHITELOCK, J. How "ready" are customers for mass customization? An exploratory investigation. European Journal of Marketing, v. 38, p. 1396-1416, 2004. http://dx.doi.org/10.1108/03090560410560164

BARE, M.; COX, J. J. Applying principles of mass customization to improve the empirical product development process. Journal of Intelligent Manufacturing, v. 19, p. 565-576, 2008. http://dx.doi.org/10.1007/s10845-008-0134-0

BARLOW, J. From craft production to mass customisation? Customer-focused approaches to housebuilding. In Proceedings of IGLC 6th Annual Conference, Sao Paulo, 1998. Disponível em: <http://www.ce.berkeley.edu/ tommelein/IGLC-6/Barlow.pdf>. Acesso em: 5 maio 2013.

BARLOW, J.; OZAKI, R. Achieving Customer Focus in Private Housebuilding: Current Practice and Lessons from Other Industries. Housing Studies, v. 18, n. 1, p. 87-101, 2003. http://dx.doi.org/10.1080/0267303032000076858.

BARLOW, J.; CHILDERHOUSE, P.; GANN, D.; HONG-MINH, S.; NAIM, M.; OZAKI, R. Choice and delivery in housebuilding: Lessons from Japan for UK housebuilders. Building research \& information, v. 31, n. 2, p. 134-145, 2003.

http://dx.doi.org/10.1080/09613210302003

BLECKER, T.; ABDELKAFI, N.; KALUZA, B.; FRIEDRICH, G. Variety steering concept for mass customization, Discussion Papers of the University of Klagenfurt, No. 2003/04, 2003. Disponível em: < http://mpra.ub.uni-muenchen.de/5251/1/MPRA_paper_5251.pdf>. Acesso em: 17 Junho 2013.

BRANDÃO, D. Q. Personalização do produto habitacional: novas demandas e respostas das tecnologias de construção. In: ENCONTRO NACIONAL DE ENGENHARIA DE PRODUÇÃO, v. 22, 2002.

CHENG, G. P.; HAN, Z. Mass Customization in the Tourism Industry of China. Advanced Materials Research, v. 683, p. 917-920, 2013. http://dx.doi.org/10.1109/WiCom.2008.2115

DAI, K.; LI, Y.; HAN, J.; LU, X.; ZHANG, S. An interactive web system for integrated threedimensional customization. Computers in Industry, v. 57, p. 827-837, 2003.

http://dx.doi.org/10.1016/i.compind.2006.04.017 
DA SILVEIRA, G.; BORENSTEIN, D.; FOGLIATTO, F. S. Mass customization: Literature review and research directions. International journal of production economics, v. 72, n. 1, p. 1-13, 2001. http://dx.doi.org/10.1016/S0925-5273(00)00079-7

DAVIS, S. M. Future perfect. MA: Addison-Wesley Publishing, 1987.

ARAUJO FILHO, J. T.; GOMES, M. D. L. B. A customização em massa na construção civil: um estudo no subsetor de edificações. Revista Produção Online, v. 10, n. 2, 2010.

DREJER, A.; GUDMUNDSSON, A. Towards multiple product development. Technovation, v. 22 , n. 12, p.733-745, 2002. http://dx.doi.org/10.1016/S0166-4972(02)00024-X

DU, X.; JIAO, J.; TSENG, M.M. Modelling platform-based product configuration using programmed attributed graph grammars. Journal of Engineering Design, v.14, n.2, p.145167, 2003. http://dx.doi.org/10.1080/0954482031000091482

DUARTE, J. P. A discursive grammar for customizing mass housing: the case of Siza's houses at Malagueira. Automation in construction, v. 14, n. 2, p. 265-275, 2005. http://dx.doi.org/10.1016/j.autcon.2004.07.013

DURAY, R.; MILLIGAN, G. W. Improving Customer Satisfaction through Mass Customization. Quality Progress, v. 32, n. 8, p. 60-66, 1999.

DURAY, R.; WARD, P. T.; MILLIGAN, G. W.; BERRY, W. L. Approaches to mass customization: configurations and empirical validation. Journal of Operations Management, v. 18, n. 6, p.605-625, 2000.http://dx.doi.org/10.1016/S02726963(00)00043-7

FRANKE, N.; PILLER, F.T. Key reserch issues in user interaction with configuration toolkits in mass customisation, The International Journal of Technology Management, v. 26, n.5, p. 578-599, 2003. http://dx.doi.org/10.1504/IJTM.2003.003424

FEITZINGER, E.; LEE, H. L. Mass customization at Hewlett-Packard: the power of postponement. Harvard Business Review, v.75, n. 1, p. 116-123, 1997.

FETTERMANN, D.C.; ECHEVESTE, M.E.S. New product development for mass customization: a systematic review. Production and Manufacturing Research, v. 2, n.1, p. 266-290, 2014. http://dx.doi.org/10.1080/21693277.2014.910715

FETTERMANN, D. C.; ECHEVESTE, M. E. S.; TEN CATEN, C.S. When and How to use the online configurator in the Automobile Industry. IEEE Latin America Transactions, v.10, n.6, p.2331-2341, 2012. http://dx.doi.org/10.1109/TLA.2012.6418140

FETTERMANN, D. C.; ECHEVESTE, M. E. S. Desenvolvimento de produto para customização em massa: alternativas para o setor de móveis modulados, Espacios, v. 32, n. $4,2011$.

FOGLIATTO, F. S.; DA SILVEIRA, G. J. Mass customization: A method for market segmentation and choice menu design. International Journal of Production Economics, 
v. 111, n.2, p. 606-622, 2008. http://dx.doi.org/10.1016/j.ijpe.2007.02.034

FOGLIATTO, F. S.; DA SILVEIRA, G. J.; BORENSTEIN, D. The mass customization decade: An updated review of the literature. International Journal of Production Economics, v.138, n.1, p. 14-25, 2012. http://dx.doi.org/10.1016/j.ijpe.2012.03.002

FRANKE, N.; PILLER, F.T. Key Research Issues in User Interaction with Configuration Toolkits in Mass Customization, International Journal of Technology Management, v.26, p. 578-599, 2003. http://dx.doi.org/10.1504/IJTM.2003.003424

FRANKE, N.; SCHREIER, M.; KAISER, U. The "I designed it myself" effect in mass customization. Management Science, v. 56, n.1, p. 125-140, 2010.

http://dx.doi.org/10.1287/mnsc.1090.1077

GIL, A. C. Como elaborar projetos de pesquisa. São Paulo, 5, 2002.

GILMORE, J. H.; PINE II, B. J. The four faces of mass customization. Harvard Business Review, v. 75, n. 1, p. 91, 1997.

GILMORE, J. H.; PINE, J. B. Markets of one: Creating customer-unique value through mass customization. Harvard Business Press, 2000.

GRENCI, R. T.; WATTS, C. A. Maximizing customer value via mass customized e-consumer services. Business Horizons, v. 50, n. 2, p.123-132, 2007.

http://dx.doi.org/10.1016/j.bushor.2006.08.006

HALMAN, J. I.; VOORDIJK, J. T.; REYMEN, I. M. Modular approaches in Dutch house building: an exploratory survey. Housing Studies, v. 23, n. 5, p. 781-799, 2008. http://dx.doi.org/10.1080/02673030802293208

HART, C. W. Mass customization: conceptual underpinnings, opportunities and limits. International Journal of Service Industry Management, v. 6, n.2, p. 36-45, 1995. http://dx.doi.org/10.1108/09564239510084932

HELANDER, M. G.; JIAO, J. Research on E-product development (ePD) for mass customization. Technovation, v. 22, n. 11, p. 717-724, 2002. http://dx.doi.org/10.1016/S0166-4972(01)00074-8

HOFMAN, E.; HALMAN, J. I.; ION, R. A. Variation in housing design: identifying customer preferences. Housing Studies, v. 21, n. 6, p. 929-943, 2006.

http://dx.doi.org/10.1080/02673030600917842

HUFFMAN, C.; KAHN, B. E. Variety for sale: mass customization or mass confusion? Journal of Retailing, v. 74, n. 4, p. 491-513, 1998. http://dx.doi.org/10.1016/S00224359(99)80105-5

JIAO, J.; TSENG, M. M. Fundamentals of product family architecture. Integrated Manufacturing Systems, v.11, n.7, p.469-483, 2000.

http://dx.doi.org/10.1108/09576060010349776 
JIAO, J.; TSENG, M. M. Customizability analysis in design for mass customization.

Computer-Aided Design, v. 36, n. 8, p. 745-757, 2004.

http://dx.doi.org/10.1016/i.cad.2003.09.012

JIAO, J.; MA, Q.; TSENG, M.M. Toward high value-added products and services: mass customization and beyond, Technovation, v. 23, n. 10, p. 809-821, 2003.

http://dx.doi.org/10.1016/S0166-4972(02)00023-8

KENDALL, S. H.; TEICHER, J. Residential Open Building, E \& FN Spon, London and New York, 2000.

KOTHA, S. From mass production to mass customization: The case of the National Industrial Bicycle Company of Japan. European Management Journal, v. 14, n. 5, p. 442-450, 1996. http://dx.doi.org/10.1016/0263-2373(96)00037-0

KRUEGER, C. Easing The Transition To Software Mass Customization. In: Software Product-Family Engineering. Springer Berlin Heidelberg, v. 2002, p. 282-293, 2001.http://dx.doi.org/10.1007/3-540-47833-7 25

KUMAR, S.; PHROMMATHED, P. New product development: an empirical study of the effects of innovation strategy, organizational learning, and market conditions. New York: Springer, 2005.

LIHRA, T.; BUEHLMANN, U.; GRAF, R. Customer preferences for customized household furniture. Journal of Forest Economics, v. 18, n. 2, p. 94-112, 2012. http://dx.doi.org/10.1016/j.jfe.2011.11.001

LIZARRALDE, G. Stakeholder participation and incremental housing in subsidized housing projects in Colombia and South Africa. Habitat International, v. 35, n. 2, p. 175-187, 2011. http://dx.doi.org/10.1016/i.habitatint.2010.08.001

MERLE, A.; CHANDON, J. L.; ROUX, E.; ALIZON, F. Perceived Value of the Mass-Customized Product and Mass Customization Experience for Individual Consumers. Production and Operations Management, v. 19, n. 5, p. 503-514, 2010. http://dx.doi.org/10.1111/j.1937-5956.2010.01131.x

MEYER, M.H.; LEHNERD, A.P. The power of product platforms: Building value and cost leadership, New York: Free Press, 1997.

MOREAU, C. P. Inviting the amateurs into the studio: Understanding how consumer engagement in product design creates value. Journal of Product Innovation Management, v. 28, n. 3, p. 409-410, 2011. http://dx.doi.org/10.1111/j.1540-5885.2011.00813.x

NAHMENS, I.; BINDROO, V. Is Customization Fruitful in Industrialized Homebuilding Industry? Journal of Construction Engineering and Management, v.137, n.12, p.10271035, 2011. http://dx.doi.org/10.1061/(ASCE)CO.1943-7862.0000396

NEVES, J. L. Pesquisa qualitativa: características, usos e possibilidades. Caderno de pesquisas em administração, v. 1, n. 3, p. 2, 1996. 
NOGUCHI, M.; HERNÀNDEZ-VELASCO, C. R. A 'mass custom design' approach to upgrading conventional housing development in Mexico. Habitat International, v.29, n. 2, p.325-336, 2005. http://dx.doi.org/10.1016/i.habitatint.2003.11.005

OGAWA, S.; PILLER, F. T. Reducing the risks of new product development. MIT Sloan Management Review, v. 47, n. 2, p. 65, 2006.

PEPPERS, D.; ROGERS, M. Enterprise One To One: Tools for Competing in the Interactive Age. Bantam Doubleday Dell Publishing Group, New York, 1997.

PILLER, F. T. Mass customization: reflections on the state of the concept. International Journal of Flexible Manufacturing Systems, v. 16, n. 4, p. 313-334, 2004. http://dx.doi.org/10.1007/s10696-005-5170-x

PINE II, J. B. Mass Customization: The New Frontier in Business Competition. Boston: Harvard Business School Press, 1993.

RIBEIRO, J.L.D.; MILAN, G.S. Entrevistas Individuais: teoria e aplicações. Porto Alegre: Fundação Empresa Escola de Engenharia da UFRGS, v.1, p.106, 2004.

RO, Y. K.; LIKER, J. K.; FIXSON, S. K. Modularity as a strategy for supply chain coordination: the case of U.S. auto. IEEE Transactions on Engineering Management, v.54, n.1, p.172-189, 2007. http://dx.doi.org/10.1109/TEM.2006.889075

ROBERT, E. B.; MEYER, M. H. Product Strategy and Corporate Success, Working paper (Sloan School of Management). Sloan School of Management, Massachusetts Institute of Technology (MIT), 1991. Disponível em $<$ http://dspace.mit.edu/bitstream/handle/1721.1/2334/SWP-323923273127.pdf?sequence=1>. Acessado em 27 agosto de 2013.

ROBERTSON, D.; ULRICH, K. Planning for product Platforms. Sloan Management Review, v.39, n. 4, p. 19-31, 1998. Disponível em <http://www.ktulrich.com/uploads/6/1/7/1/6171812/platforms-smr.pdf $>$. Acessado em 10 de julho de 2012.

ROCHA, C.G.; FORMOSO, C. T.; SANTOS, A. An Overview Of The Customisation Strategies Developed By Four Organisations Of The House-Building Sector. In: 20th Conference Of The International Group For Lean Construction, 2012, San Diego. Proccedings of the 20th Conference of the International Group for Lean Construction, 2012.

ROYER, R. Implantação da customização em massa na estratégia da manufatura. In: . Anais do XXVII Encontro Nacional De Engenharia De Produção, 2007, Foz Do Iguaçu. Disponível em: <http://www.abepro.org.br/biblioteca/ENEGEP2007_TR630469_9153.pdf>. Acesso em:18 jul 2013.

SALVADOR, F.; FORZA, C.; RUNGTUSANATHAM, M. Modularity, product variety, production volume, and component sourcing: theorizing beyond generic prescriptions. Journal of Operations Management, v. 20, n. 5, p. 549-575, 2002. http://dx.doi.org/10.1016/S0272-6963(02)00027-X 
SALVADOR, F.; FORZA, C. Configuring products to address the customizationresponsiveness squeeze: a survey of management issues and opportunities. International Journal of Production Economics, v. 91, n. 3, p. 273-291, 2004. http://dx.doi.org/10.1016/i.ijpe.2003.09.003

SHIN, Y.; AN, S.; CHO, H.; KIM, G.; KANG, K. Application of information technology for mass customization in the housing construction industry in Korea. Automation in Construction, $\mathrm{v}$. 17, n. 7, p. 831-838, 2008. http://dx.doi.org/10.1016/j.autcon.2008.02.010

SIEVÄNEN, M. What Is Customization? In: 9th International Annual Conference Of European Operations Management Association, Copenhagen, Denmark, 2002. Disponível em <http://webhotel2.tut.fi/cmc/pdf/what-is-customization.pdf $>$. Acessado em 01 de junho de 2013.

SIMPSON, T. W. Product platform design and customization: status and promise. AI EDAM: Artificial Intelligence for Engineering Design, Analysis and Manufacturing, v. 18, n. 1, p. 3-20, 2004. http://dx.doi.org/10.10170S0890060404040028.

SQUIRE, B.; READMAN, J.; BROWN, S.; BESSANT, J. Mass customization: the key to customer value? Production Planning \& Control: The management of Operations, v. 15, n. 4, p. 459-471, 2004. http://dx.doi.org/10.1080/0953728042000238755

TSENG, M. M.; JIAO, R. J.; WANG, C. Design for mass personalization. CIRP AnnalsManufacturing Technology, v. 59, n. 1, p. 175-178, 2010.

http://dx.doi.org/10.1016/i.cirp.2010.03.097

TSENG, M. M.; JIAO, J. Design for mass customization. CIRP Annals-Manufacturing Technology, v.45, n. 1, p. 153-156, 1996. http://dx.doi.org/10.1016/S00078506(07)63036-4

TSENG, M.M.; JIAO, J. Mass Customization. In. Handbook of Industrial Engineering: Technology and Operations Management, Salvendy, G. (Ed). $3^{\mathrm{a} e d .}$ New York: John Wiley \& Sons, 2007.

TU, Y. L.; XIE, S. Q.; FUNG, R. Y. Product development cost estimation in mass customization. IEEE Transactions on Engineering Management, v. 54, n. 1, p. 29-40, 2007. http://dx.doi.org/10.1109/TEM.2006.889065

ULRICH, K. The role of product architecture in the manufacturing firm. Research Policy,v.24, n.3, p. 419-440, 1995. http://dx.doi.org/10.1016/0048-7333(94)00775-3

ULRICH, K. T. Design, Creation Of Artifacts In Society: Utility And Complexity, University of Pennsylvania, 2011. Disponível em $<$ http://opim.wharton.upenn.edu/ ulrich/designbook.html>. Acessado em 10 de julho de 2013.

VIDOR, G.; MEDEIROS, J.F.; RIBEIRO, J.L.D. Modelo de Classificação de Características de Customização em Massa para Serviços. Revista Produção Online, Florianópolis, SC, v.13, n. 3, p. 974-1001, 2013. http://dx.doi.org/10.14488/1676-1901.v13i3.1333 
VOSS, C. A.; HSUAN, J. Service architecture and modularity. Decision Sciences, v.40, n.3, p.541-569, 2009. http://dx.doi.org/10.1111/j.1540-5915.2009.00241.x

ZHANG, M.; CHEN, Y.J.; TSENG, M.M. Distributed knowledge management for product and process variety in mass customisation. International Journal of Computer Applications in Technology, v.23, n.1, p.13-30, 2005. http://dx.doi.org/10.1504/IJCAT.2005.006419

ZEITHAML, V. A. Consumer perceptions of price, quality, and value: a means-end model and synthesis of evidence. Journal of Marketing, v.52, p.2-22, 1988.

http://dx.doi.org/10.2307/1251446

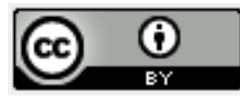

Artigo recebido em 28/01/2014 e aceito para publicação em 12/11/2014

DOI: http://dx.doi.org/ 10.14488/1676-1901.v15i1.1728

Revista Produção Online, Florianópolis, SC, v.15, n. 1, p. 135-162, jan./mar. 2015. 
ANEXO A - Questionário Qualitativo para Empresas da Construção Civil que aplicam a estratégia de Customização em Massa

\begin{tabular}{|l|l|}
\hline & IDENTIFICAÇÃO \\
\hline Identificação do aplicador:_ data: & Cargo do Entrevistado: \\
\hline Construtora: & do questionário:
\end{tabular}

\section{Dê sua opinião em relação a estas questões:}

Qual a quantidade de funcionários no processo construtivo? E na área de gestão?

Qual o tempo de atuação da construtora?

No momento, quantos empreendimentos a construtora tem?

Atua em alguma outra região? E cidade?

Qual o número de pavimentos do empreendimento?

Qual o número de andares de todos os pavimentos? E quantas unidades são por andar?

Em sua opinião, como classificaria a customização oferecida pela construtora? Semi-Customizada ou Customizada?

O ponto de desacoplamento (DP) é o ponto, no processo produtivo onde as especificações do cliente são consideradas e onde começa, de forma efetiva, a customização do produto. Em qual etapa encontra-se o DP da construtora?

Aquisição do Terreno

Elaboração dos Projetos Padronizados

Locação da Fundação

Execução da Infraestrutura

Execução da Superestrutura (pilares e lajes)

Demarcação da Alvenaria (Layout das Plantas)

Tubulação das Instalações

Tubulação Hidros sanitária

Tubulação de Drenagem dos Split's

Fiação dos Apartamentos

Instalação de gás

Assentamento de Revestimento

Forro de Gesso

Piso Cimentado de regularização

Assentamento de Revestimento (piso e rodapé)

Aplicação de Bancada

Aplicação de filetes de granito no piso dos box

Pintura (Parede e Teto)

Aplicação dos Tanques nas áreas de serviços

Assentamentos das Portas dos apartamentos

Instalação de louça sanitária

Instalação dos metais sanitários

Instalação de Chuveiros

Instalação de Disjuntores e Espelhos

1 Quais e quantas são as opções que são dadas para o cliente escolher em cada empreendimento? (Acabamentos, Paredes, Pisos, Quantidade de Quartos)

1 Como são definidas estas opções citadas anteriormente? Há alguma ferramenta ou método para isto?

1 Em sua opinião, existe a necessidade de uma maior quantidade de opções de produtos para atender as demandas dos clientes? Que tipos de opções deveriam ser incorporadas?

1 Em relação a serviços, os clientes solicitam algum tipo de serviço que a construtora não pode atender? Que tipos de serviços deveriam ser incorporados?

1 A empresa venderia mais se pudesse ou oferecesse mais alternativas? Vale a pena incluir estas opções na empresa?

1 Em relação aos serviços e produtos que pode haver na pós-ocupação da habitação? Tais como, troca de alvenarias, acabamentos, iluminação? A empresa oferece suporte e opções para esta personalização?

1 Quais solicitações ou tipo de solicitações dos clientes não costumam ser atendidas pela empresa? Por quais razões?

2 Quais escolhas de opções de produtos são mais frequentes pelos clientes?

2 Como é feita a escolha das opções pelo cliente? Existe alguma dificuldade ou problema durante este processo?

Revista Produção Online, Florianópolis, SC, v.15, n. 1, p. 135-162, jan./mar. 2015. 


\section{Vendedor}

Interface com o computador

Projeto interativo entre cliente-projetista

Cite outra

2 Qual o auxílio-orientação que é dado ao cliente para a escolha das alternativas dadas?

2 Na sua opinião, a disponibilização de um programa que permita o cliente simular como seriam as alterações seria útil para a empresa?

3 Qual o procedimento de transferência dos dados do pedido do cliente para a construção?

3 Quais são os meios utilizados para esta transferência?

3 Houve ou há problemas/erros relacionados à transferência destes pedidos?

3 Quando o cliente opta por alguma alternativa que não está prevista no catálogo de opções, como este processo é realizado?

4 Quais são as principais dificuldades encontradas para atender a variedade das necessidades de customização dos clientes?

4 Quais os setores funcionais da empresa são mais envolvidos neste processo?

4 Há alguma tecnologia de manufatura para auxiliar este processo de customização?

4 Para as habitações customizadas, como é organizada e programada a produção? Há alguma diferença das padronizadas?

4 A construtora aplica algum destes processos ou metodologias? De alguma forma elas auxiliam a empresa a atender a demanda customizada?

Manufatura ágil (agile manufacturing),

Produção enxuta (lean production)

Projeto e manufatura direcionadas ao cliente

Gerenciamento da cadeia de suprimentos (supply chain management)

Processo de desenvolvimento de produto

Plataforma de produto

Modularização

Cite outras

4 Há diferença de prazos para entrega de uma unidade customizada e uma padronizada? Justifique.

5 Como ocorre a construção e entrega da unidade customizada para o cliente? Há algum tipo de acompanhamento deste processo?

5 A empresa acompanha a utilização, reutilização ou alteração da unidade habitacional após a entrega?

5 Existe algum produto/serviço disponibilizado pela empresa posterior ao processo de venda e entrega?

\section{OPINIÃO SOBRE A CUSTOMIZAÇÃO}

Em sua opinião, quais as VANTAGENS de optar pelo serviço de customização?

Em sua opinião, quais as DESVANTAGENS de optar pelo serviço de customização?

\section{Observações}

Revista Produção Online, Florianópolis, SC, v.15, n. 1, p. 135-162, jan./mar. 2015. 